\title{
Synthesis, Docking and Antimicrobial Activity Studies of Some Novel Fused Thiophenes of Biological Interest
}

\author{
Anitha Ramalingam ${ }^{1 *}$, Janardhanan Sarvanan ${ }^{2}$ \\ 'School of Pharmaceutical Sciences, Dayananda Sagar University, Kumaraswamy Layout, Bangalore, Karnataka, INDIA. \\ ${ }^{2}$ Faculty of Pharmaceutical Sciences, PES University, HN Campus, Bangalore, Karnataka, INDIA.
}

\begin{abstract}
Objective: Thiophenes are a part of many natural and synthetic biologically active products displaying several pharmacological activities. Area of antibacterial and antifungal research is still on because of faster development of drug resistance of existing molecules by the microbes. Methods: The starting material 2 -amino-3-carbethoxy-6-N- methyl piperidino thiophene[1] was synthesized by adapting a well-known and versatile Gewald's reaction with excellent yield (85-98\%). The title compounds (1 a-l) were obtained by treating the starting material[1] with various arylaldehydes, as per Scheme 1. Structures of the synthesized compounds were confirmed by physical (Lassaigne's test, MP and $R_{f}$ vaiues) and spectral data (IR, UV, NMR, Mass and CHN Analyses) and all the new compounds were studied for molecular docking and screened for antimicrobial activity. Results: Among the new compounds, the compounds $1 \mathrm{f}, 1 \mathrm{j}$ and $1 \mathrm{l}$ exhibited good activity against pathogenic bacteria and fungi. Discussion The aim of designing and
\end{abstract}

synthesizing new molecules as antimicrobials is because of predominant resistance of bacteria and fungi against the existing drugs using Gewald's and Schiff's Reaction. Conclusion: It is concluded that some of the new piperidino thiophene analogues reported here displayed promising activity. Key words: Synthesis, Docking, Piperidino thiophene, Gewald's reaction, Antimicrobial activity.

Correspondence

Mrs. Anitha Ramalingam,

School of Pharmaceutical Sciences, Dayananda Sagar University, Kumaraswamy Layout, Bangalore-560111, Karnataka, INDIA.

Phone: +919902559552

Email: anitramar16@gmail.com

DOI: 10.5530/jyp.2020.12.24

\section{INTRODUCTION}

Thiophene based pharmaceuticals are rapidly becoming an important class of therapeutic agents and are likely to replace many existing pharmaceuticals in the near future. They are reported to possess, diverse pharmaceutical applications such as anti-inflammatory agents, ${ }^{1}$ Antifungal, ${ }^{2}$ Antimicrobial, ${ }^{3,4}$ allosteric enchancers, ${ }^{5,6}$ antibacterial, ${ }^{7-10}$ Among the antimicrobial agents, thiophene derivatives are known to have a promising biological activity like Cephalothin(1), Cephalorodine(2), Teconazole(3) etc.

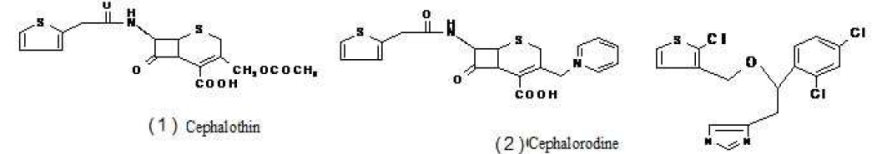

(3) Teconazole

Many condensed Thiophenes are synthesized ${ }^{3-10}$ and reported to be antimicrobial and few among them are presented below: Saravanan et al. ${ }^{4}$ (1997) synthesized some benzo(b)thiophenes (4) as potential antimicrobial agents. Saravanan et al. ${ }^{7}(2003)$ have reported the synthesis, characterization and antibacterial activity of some Schiff bases of 2-amino-3-( $N$-tolyl carboxamido)-4, 5-pentamethylene thiophenes (5). Sara Tehranchian et al. ${ }^{9}$ (2005) reported the synthesis and antibacterial activity of 1- (1,2,4-triazol-3-yl) and 1-(1, 3, 4-thiadiazol-2-yl)-3methylthio-6,7-dihydro-benzo(C)thiophen-4(5H)ones(6,7,8). Mohan et al. ${ }^{9}$ (2003) reported the synthesis, characterization of some schiff bases of 2-Amino-3- (N-tolylcarboxamido)-4,5,6,7-tetrahydro benzol(b) thiophenes (9).

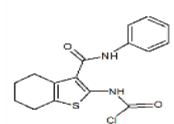

(4)

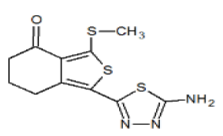

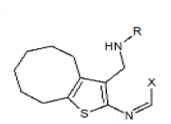

(5)

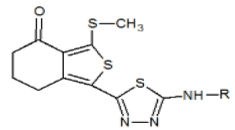

(6)

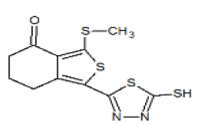

(7)
(8)<smiles>CC=Nc1sc2c(c1C(N)=O)CCCC2</smiles>

(9)

Various condensed and substituted Thiophenes ${ }^{11}$ are being synthesized either by adapting Gewald ${ }^{12}$ or Paal-Knorr reactions ${ }^{13,14}$ in which a sulfur atom is introduced using elemental sulfur, $\mathrm{H}_{2} \mathrm{~S}$, Lawesson's reagent, phosphorus pent sulfide, or bis-(trimethylsilyl)sulfide. ${ }^{15}$ The above observations encouraged us to take up the synthesis of novel, stable, cost effective and biologically active thiophenes with the presumption that these compounds could be potential agents for antimicrobial activity with fewer adverse effects. To the best of our knowledge, there has been no report available on the synthesis of fused piperidino thiophenes, which were synthesized in the present study and in the open literature so far.

\section{MATERIALS AND METHODS}

Melting points are uncorrected; the UV spectra were recorded on Shimadzu 1601 spectrometer, IR (KBr) were recorded on Perkin-Elmer FT-IR 1600 spectrometer, $\mathrm{H}^{1}$ NMR spectra were recorded on Brucker AMX 400 using the solvent $\left(\mathrm{CDCl}_{3} 7.26 \mathrm{ppm}\right.$ and $77.0 \mathrm{ppm}$, DMSO-d6 $2.49 \mathrm{ppm}$ and $39.7 \mathrm{ppm}$ ) and TMS used as an internal standard. Lowresolution MS data were obtained using ESI and high-resolution spectra were recorded on QSTARXL hybrid MS/MS system Elemental 
analyses were within $\pm 0.4 \%$ of their calculated values. For Molecular Docking, the ligand structures are built by using builder in Molinspiration Cheminformatics and converted to 3D with Corina3D. The synthesized molecules (ligands) were docked into the active site using Molecular docking software PATCH Dock with default parameters. The precise location of the binding site and the potentiality of the ligand to bind to the active site were determined using an automated docking software, molegro virtual docker 2008, version 3.2.1 (Molegro ApS, Aarhus, Denmark, http://molegro.com).

\section{Methods}

\section{Synthesis}

\section{STEP 1: Methodology- Gewald's Reaction}

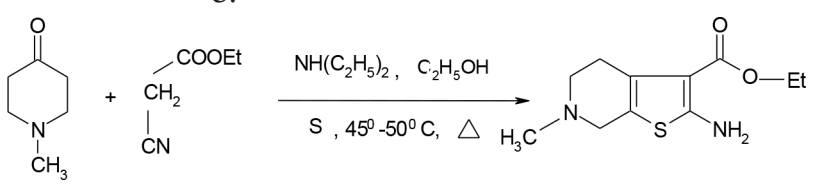

N-methyl piperidone Ethylcyanoacetate 2-amino, 3-carbethoxy, 6-N-methylpiperidinothiophene

Synthesis of 2 -amino-3-carbethoxy-6-N- methyl piperidino thiophene [1]:

This synthetic procedure is based on Gewald synthesis. This is the usual route to synthesize 2 -amino thiophenes. It consists of the base catalyzed condensation of a ketone having an active $-\mathrm{CH}_{2}$ - group, with a ketonitrile to form an olefin, followed by cyclization with the elemental Sulphur.

\section{Procedure}

A mixture of N-methyl piperidin-4-one (a) and Ethyl cyano acetate (b) (0.04 mole each), $40 \mathrm{ml}$ alcohol and Sulphur (1.28gms 0.04 mole) were taken in a $100 \mathrm{ml}$ conical flask and the mixture was stirred on a hot plate (magnetic stirrer) till the temperature reached $45-50^{\circ} \mathrm{C}$. $4 \mathrm{ml}$ of Diethylamine was added drop wise till the Sulphur goes into the solution. Then the mixture was cooled overnight to get the desired parent compound 1 as crystals. Purified by recrystalization using ethanol. The formation of the new piperidino thiophene 1 was confirmed by Lassaignes test, TLC, UV and IR spectra. The melting point of the parent compound 1 was found to be $103-105^{\circ} \mathrm{C}$. The IR spectra exhibit a distinct peak at $3339 \mathrm{~cm}^{-1}\left(\mathrm{NH}_{2}\right.$ group) confirms the formation of the parent compound 1 .

STEP 2: Methodology- Schiff's Reaction

Scheme 1: Synthesis of 2 -((substituted benzylidene) -imino)-3carbethoxy-6-N- methyl piperidino thiophene analogues (1a-1).

General procedure for the synthesis of $1 \mathrm{a}-1$

An equimolar mixture of (0.005 mole) of the parent compound 1 (2 -amino-3-carbethoxy-6-N- methyl piperidino thiophene) and the desired aryl aldehydes ( $\mathrm{a}-\mathrm{l}$ ) in $30 \mathrm{ml}$ of ethyl alcohol with few drops of glacial acetic acid was refluxed for one hour, then the mixture was cooled to obtain the crystals of the desired title compounds. (the optimization of the solvent is shown in Table 1) The title compounds were purified by recrystalization using DMF: Water mixture (5:1 ratio). The formation of the title compounds were preliminarily confirmed by the difference in $\mathrm{MP}, \mathrm{R}_{f}$ value, UV spectra(bathochromic shift) and specific IR peak where there is absence of the primary aromatic amino peak at $3339 \mathrm{~cm}^{-1}$ and an appearance of a new peak at $1550-1560 \mathrm{~cm}^{-1}$ for $-\mathrm{N}=\mathrm{CH}$ - (imine gp)in the title compounds compared to the parent compound. The pure title compounds were also characterized by NMR and Mass and the values are recorded in Table 2.

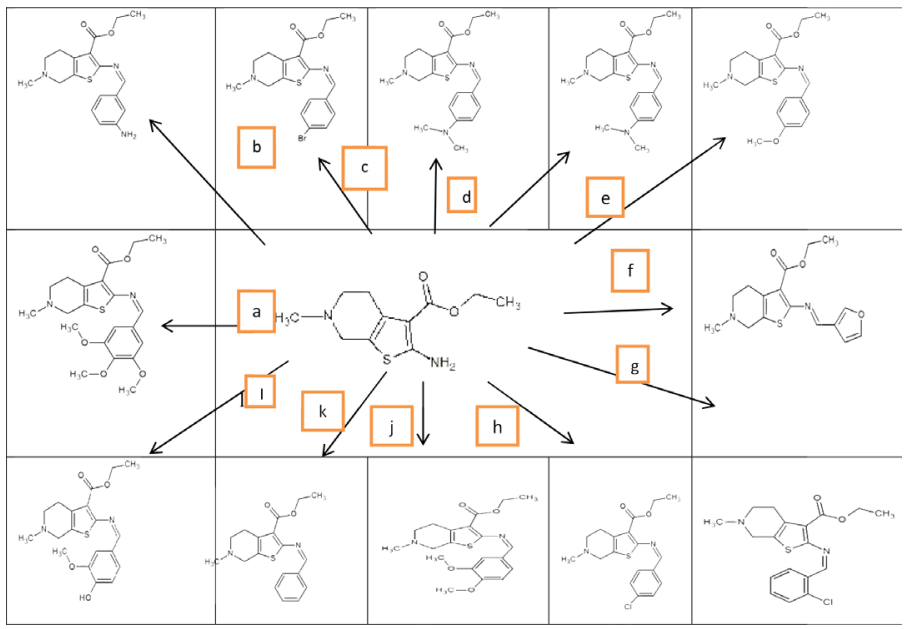

1. Antimicrobial activity by Kirby Baurer Method

\section{Method:}

- The in vitro antibacterial assay was carried out by adopting the Kirby Baurer method for aerobic testing.

- The microbe tested was sub-cultured twice on Mueller-Hinton agar and the colonies (5-7) were then transferred aseptically into individual tubes containing sterile nutrient broth $(10 \mathrm{ml})$.

- The tubes were incubated for a period of $8-12 \mathrm{hrs}$ at $37^{\circ} \mathrm{C}$ to attain growth at $\log$ phase.

- Subsequently, these inoculates were diluted with sterile distilled water to obtain a density corresponding approximately to 0.5 McFarland standard turbidity scale $\left(1 \times 10^{6} \mathrm{cfu} / \mathrm{ml}\right)$.

- $100 \mu \mathrm{l}$ of the test organism is aseptically transferred to the sterile nutrient agar plates (each plate containing $20-25 \mathrm{ml}$ of the sterile medium) and spread plate technique was followed using a sterile L-shaped glass rod.

Table 1: Optimization of solvent, base, catalyst for the synthesis of compounds (1a).

\begin{tabular}{|c|c|c|c|c|c|c|c|}
\hline \multirow{2}{*}{ S.No. } & \multirow{2}{*}{ Entry Starting } & \multirow{2}{*}{ Compound } & \multirow{2}{*}{$\begin{array}{l}\text { Catalyst material } \\
\text { (Benzaldehyde) }\end{array}$} & \multirow[t]{2}{*}{ Solvent } & \multicolumn{3}{|c|}{ Condition Time Yield } \\
\hline & & & & & Ns & (h) & (\%) \\
\hline 1 & 1 & 2 & AcOH (0.1 eq.) & $\mathrm{CH}_{2} \mathrm{Cl}_{2}$ & reflux & 1 & 88 \\
\hline 2 & 1 & 2 & $\mathrm{AcOH}$ (0.1 eq.) & $\mathrm{CHCl}_{3}$ & reflux & 1 & 90 \\
\hline 3 & 1 & 2 & AcOH (0.1 eq.) & $\mathrm{EtOH}$ & reflux & 1 & 93 \\
\hline 4 & 1 & 2 & $\mathrm{AcOH}$ (0.1 eq.) & $\mathrm{CH}_{3} \mathrm{CN}$ & reflux & 1 & 82 \\
\hline 5 & 1 & 2 & $\mathrm{AcOH}$ (0.1 eq.) & DMF & reflux & 1 & 70 \\
\hline 6 & 1 & 2 & $\mathrm{AcOH}$ (0.1 eq.) & DMSO & reflux & 1 & 50 \\
\hline
\end{tabular}

The compound 1 was converted to the corresponding Schiff bases by using ethanol as solvent with catalytic amount of acetic acid at reflux temperature for 1-2 $\mathrm{h}$ gave in $85 \%$ to $98 \%$ yield, (1a-1) in Scheme 1 . 
Table 2: Spectral Details of synthesized analogs of 2 substituted amino-3-carbethoxy-6-N- methyl piperidino thiophene (1a-I).
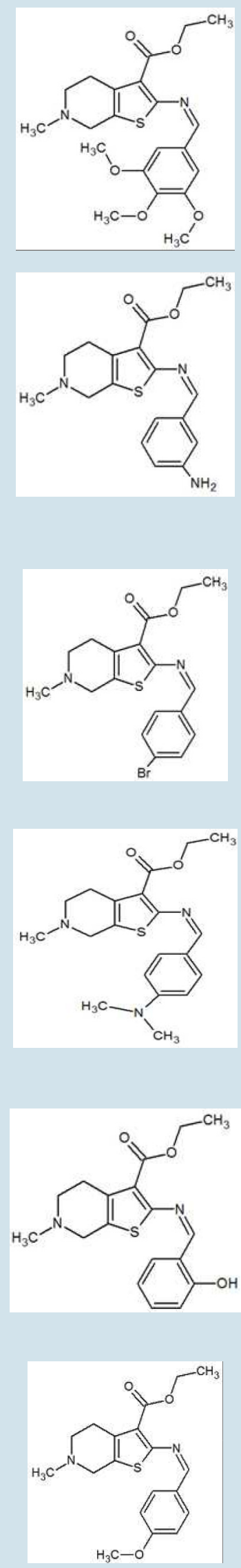

\section{S1-3(a)}

Molecular Mass 418.16

HNMR data (ppm)

$\mathrm{H} 1: \delta 1.32(\mathrm{~S}), \mathrm{H} 2$ 22.27-2.69(d), $\mathrm{H} 3 \delta 3.73(\mathrm{~m}), \mathrm{H} 4: \delta 4.29(\mathrm{~s}), \mathrm{H} 5: \delta 6.63(\mathrm{~s})$, H6: $\delta 7.02(\mathrm{~d}), \mathrm{H} 7 \delta 8.13(\mathrm{~d})$

S1-3(b)

Molecular Mass 343.44

HNMR data (ppm)

$\mathrm{H} 1: \delta 1.30(\mathrm{~S}), \mathrm{H} 2 \delta 2.27(\mathrm{~S})$

$\mathrm{H} 32.69(\mathrm{~d}), \mathrm{H} 4: \delta 3.62(\mathrm{~d})$,

H5: $84.09(\mathrm{~d}), \mathrm{H} 6: \delta 4.29$ (d),

$\mathrm{H} 7: \delta 6.53(\mathrm{~d}), \mathrm{H} 8: \delta 7.46(\mathrm{~d})$,

$\mathrm{H9:88.15( \textrm {s } )}$

S1-3(c)

Molecular Mass 407.33

HNMR data (ppm)

$\mathrm{H} 1: \delta 1.30(\mathrm{~S}), \mathrm{H} 2 \delta 2.27(\mathrm{~S}) \mathrm{H} 3: \delta 2.62$ (d), H4: $\delta 3.6$ 5(d),H5: $44.26(\mathrm{~S}), \mathrm{H} 6: \delta 7.58(\mathrm{~m}), \mathrm{H} 7: 8.15(\mathrm{~s})$

S1-3(d)

Molecular Mass 371.50

HNMR data (ppm)

$\mathrm{H} 1: \delta 1.30(\mathrm{~S}), \mathrm{H} 2$ $\delta 2.27$ (S) H3: $\delta 2.62(\mathrm{~d})$, $\mathrm{H} 4: \delta 2.812(\mathrm{~d})$

,H5: $83.65(\mathrm{~d}), \mathrm{H} 6: \delta 4.29(\mathrm{~S}), \mathrm{H7}: \delta 6.65$ (d)H8:

$\delta 7.41-\delta 7.62(\mathrm{~m}), \mathrm{H} 9: \delta 8.15(\mathrm{~s})$

S1-3(e)

Molecular Mass 344.43

HNMR data (ppm)

$\mathrm{H} 1: \delta 1.30(\mathrm{~S}), \mathrm{H} 2$ $\delta 2.27-2.69(\mathrm{~d}), \mathrm{H} 3$ $\delta 3.65-3.73(\mathrm{~m}), \mathrm{H} 4: \delta 4.29(\mathrm{~d}), \mathrm{H} 5: \delta 6.85(\mathrm{~d})$ $\mathrm{H} 6: \delta 7.58(\mathrm{~d}), \mathrm{H} 7$ 88.16(d)

S1-3(f)

Molecular Mass 358.46

HNMR data (ppm)

$\mathrm{H} 1: \delta 1.30(\mathrm{~S}), \mathrm{H} 2$ $\delta 2.27-2.69(\mathrm{~d}), \mathrm{H} 3$

$\delta 3.653 .73(\mathrm{~m}), \mathrm{H} 4: \delta 4.29(\mathrm{~d}), \mathrm{H} 5: \delta 6.85(\mathrm{~d})$

$\mathrm{H} 6: \delta 7.58(\mathrm{~d})$

$\mathrm{H} 7: \delta 8.16(\mathrm{~d})$

\section{Chemical / IUPAC Name}

2-[(3,4,5-trimethoxy-benzylidene)- amino]-6-methyl-4,5,6,7tetrahydro- thieno[2,3-c]pyridine-3-carboxylic acid ethyl ester

Infrared Spectroscopy Values

C-H (str): $2996.26 \mathrm{~cm}^{-1}, \mathrm{H}=\mathrm{C}$ (str): $3035.65 \mathrm{~cm}^{-1}$

$\mathrm{C}=\mathrm{N}$ (str): $2278.16 \mathrm{~cm}^{-1}, \mathrm{C}-\mathrm{O}$ (str): $1184.23 \mathrm{~cm}^{-1}$

$\mathrm{C}=\mathrm{O}(\mathrm{str}): 1738.16 \mathrm{~cm}^{-1}, \mathrm{C}-\mathrm{S}$ (Str): $2589.54 \mathrm{~cm}^{-1}$

\section{Chemical / IUPAC Name}

2-[(2-amino-benzylidene)-amino]-6- methyl-4,5,6,tetrahydrothieno[2,3-c]pyridine-3-carboxylic acid ethyl ester

\section{Infrared Spectroscopy Values}

C-H (str): $2989.42 \mathrm{~cm}^{-1}, \mathrm{H}=\mathrm{C}$ (str): $3036.71 \mathrm{~cm}^{-1}$ $\mathrm{N}-\mathrm{H}(\mathrm{Str}): 3448.59 \mathrm{~cm}^{-1}, \mathrm{C}=\mathrm{N}$ (str): $2268.55 \mathrm{~cm}^{-1}$ C-O (str): $1171.33 \mathrm{~cm}^{-1}, \mathrm{C}-\mathrm{N}(\mathrm{Str}): 1286.82 \mathrm{~cm}^{-1}$ $\mathrm{N}-\mathrm{H}\left(\right.$ Ben): $1621.29 \mathrm{~cm}^{-1}, \mathrm{C}=\mathrm{O}(\mathrm{str}): 1736.19 \mathrm{~cm}^{-1}$ C-S (Str): $2569.48 \mathrm{~cm}^{-1}$

Chemical / IUPAC Name

2-[(p-bromo-benzylidene)-amino]-6- methyl-4,5,6tetrahydrothieno[2,3-c]pyridine-3-carboxylic acid ethyl ester

Infrared Spectroscopy Values

C-H (str): $2928.76 \mathrm{~cm}^{-1}, \mathrm{H}=\mathrm{C}$ (str): $3059.51 \mathrm{~cm}^{-1}$ $\mathrm{C}=\mathrm{N}$ (str): $2238.22 \mathrm{~cm}^{-1}, \mathrm{C}-\mathrm{OH}(\mathrm{str}): 3312.45 \mathrm{~cm}^{-1}$ C-O (str): $1179.66 \mathrm{~cm}^{-1}, \mathrm{C}=\mathrm{O}(\mathrm{str}): 1739.29 \mathrm{~cm}^{-1}$ C-S (Str): $2565.11 \mathrm{~cm}^{-1}$

Chemical / IUPAC Name

2-[(4 dimethylamino-benzylidene)- amino]-6-methyl-4,5,6,7tetrahydro- thieno[2,3-c]pyridine-3-carboxylic acid ethyl ester

Infrared Spectroscopy Values

C-H (str): $2982.52 \mathrm{~cm}^{-1}, \mathrm{H}=\mathrm{C}$ (str): $3032.65 \mathrm{~cm}^{-1}$ $\mathrm{N}-\mathrm{H}\left(\right.$ Str): $3241.42 \mathrm{~cm}^{-1}, \mathrm{C}=\mathrm{N}$ (str): $2265.25 \mathrm{~cm}^{-1}$ C-O (str): $1171.33 \mathrm{~cm}^{-1}, \mathrm{C}-\mathrm{N}(\mathrm{Str}): 1216.54 \mathrm{~cm}^{-1}$ $\mathrm{N}-\mathrm{H}($ Ben $): 1620.27 \mathrm{~cm}^{-1}, \mathrm{C}=\mathrm{O}$ (str):1736.19 $\mathrm{cm}^{-1}$ C-S (Str): $2573.44 \mathrm{~cm}^{-1}$

Chemical / IUPAC Name

2-[(2-Hydroxy-benzylidene)-amino]-6- methyl-4,5,6,7- tetrahydrothieno[2,3-c]pyridine-3-carboxylic acid ethyester

Infrared Spectroscopy Values

C-H (str): $2915.36 \mathrm{~cm}^{-1}, \mathrm{H}=\mathrm{C}$ (str): $3052.25 \mathrm{~cm}^{-1}$ $\mathrm{C}=\mathrm{N}$ (str): $2255.16 \mathrm{~cm}^{-1}, \mathrm{C}-\mathrm{OH}(\mathrm{str}): 3314.12 \mathrm{~cm}^{-1}$ $\mathrm{C}-\mathrm{O}$ (str): $1173.63 \mathrm{~cm}^{-1}, \mathrm{C}=\mathrm{O}(\mathrm{str}): 1725.29 \mathrm{~cm}^{-1}$ C-S (Str): $2552.64 \mathrm{~cm}^{-1}$

Chemical / IUPAC Name

2-[(4-methoxy-benzylidene)-amino]-6- methyl-4,5,6,7-tetrahydrothieno[2,3-c]pyridine-3-carboxylic acid ethylester

Infrared Spectroscopy Values

C-H (str): $2915.36 \mathrm{~cm}^{-1}, \mathrm{H}=\mathrm{C}$ (str): $3052.25 \mathrm{~cm}^{-1}$

$\mathrm{C}=\mathrm{N}(\mathrm{str}): 2255.16 \mathrm{~cm}^{-1}, \mathrm{C}-\mathrm{O}(\mathrm{str}): 1184.63 \mathrm{~cm}^{-1}$

$\mathrm{C}=\mathrm{O}(\mathrm{str}): 1735.29 \mathrm{~cm}^{-1}, \mathrm{C}-\mathrm{S}(\mathrm{Str}) 2569.44 \mathrm{~cm}^{-1}$

Continued... 
Table 2: Cont'd.
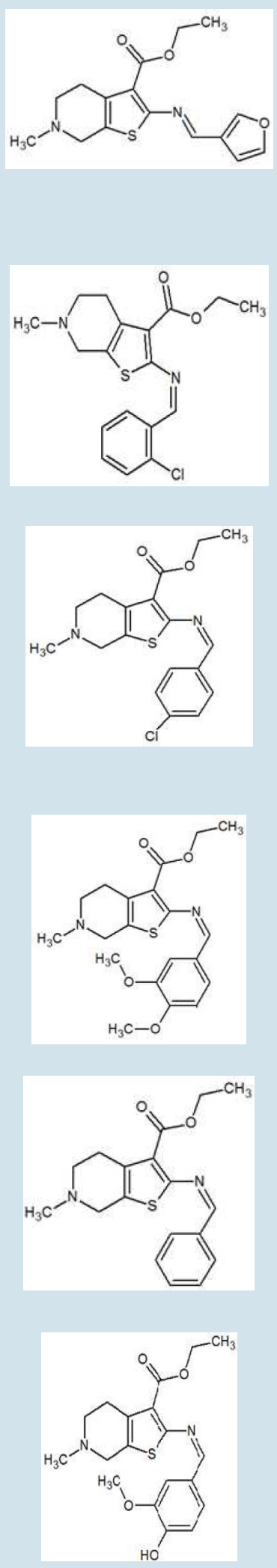

S1-3(g)

Molecular Mass 358.46

HNMR data (ppm)

$\mathrm{H} 1: \delta 1.30(\mathrm{~S}), \mathrm{H} 2$ $\delta 2.27(\mathrm{~d}) \mathrm{H} 3: \delta 2.69(\mathrm{~m})$, $\mathrm{H} 4: \delta 3.63 .73(\mathrm{~m})$,

H5: $\delta 4.29(\mathrm{~d}), \mathrm{H} 6: \delta 6.35(\mathrm{~d})$, $\mathrm{H} 7: \delta 7.42-\delta 7.58(\mathrm{~m}$

S1-3(h)

Molecular Mass 362.87

HNMR data (ppm)

H1: $1.30(\mathrm{~S}), \mathrm{H} 2$ $82.27-2.69(\mathrm{~d}), \mathrm{H} 3$ $\delta 3.65-3.73(\mathrm{~m}), \mathrm{H} 4: \delta 4.29(\mathrm{~d}), \mathrm{H} 5: \delta 6.85(\mathrm{~d})$ H6: $7.32(\mathrm{~d}), \mathrm{H} 7: \delta 7.58(\mathrm{~d}), \mathrm{H} 8: \delta 8.16(\mathrm{~d})$

S1-3(i)

Molecular Mass 362.09

HNMR data (ppm)

H1: $\delta 1.30(\mathrm{~S}), \mathrm{H} 2$ $\delta 2.27-2.69(\mathrm{~d}), \mathrm{H} 3 \delta 3.65$ 3.73(m), H4: $84.29(\mathrm{~d}), \mathrm{H} 5: \delta 6.85(\mathrm{~d})$

H6: $\delta 7.32(\mathrm{~d}), \mathrm{H} 7: \delta 7.58(\mathrm{~d}), \mathrm{H} 8: \delta 8.16(\mathrm{~d})$

\section{S1-3(j)}

Molecular Mass 388.15

HNMR data (ppm)

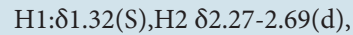

$\mathrm{H} 383.73(\mathrm{~m}), \mathrm{H} 4: \delta 4.29(\mathrm{~d})$,

$\mathrm{H} 5: \delta 6.79(\mathrm{~s}) \mathrm{H} 6: \delta 7.15(\mathrm{~d})$,

H7 $88.16(\mathrm{~d})$

S1-3(k)

Molecular Mass 328.43

HNMR data (ppm)

$\mathrm{H} 1: \delta 1.30(\mathrm{~S}), \mathrm{H} 2 \delta 2.27(\mathrm{~S})$

H3: $\delta 2.62(\mathrm{~d}), \mathrm{H} 4: \delta 3.65(\mathrm{~d})$,

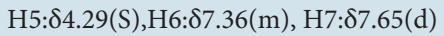
$\mathrm{H} 8: 8.15(\mathrm{~s})$

S1-3(1)

Molecular Mass 374.46

HNMR data (ppm)

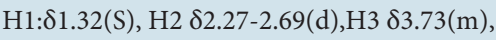
H4: $\delta 4.29(\mathrm{~s}), \mathrm{H} 5 \delta 5.06(\mathrm{~s}), \mathrm{H} 6: \delta 6.72(\mathrm{~s}) \mathrm{H} 7$ : $\delta 7.02(\mathrm{~d}), \mathrm{H} 8$ 88.13(s)

\section{Chemical / IUPAC Name}

2-[(Furan-3-ylmethylene)-amino]-6-methyl-4,5,6,7-tetrahydrothieno[2,3-c] pyridine-3-carboxylic acid ethyl ester

Infrared Spectroscopy Values

C-H (str): $2982.52 \mathrm{~cm}^{-1}, \mathrm{H}=\mathrm{C}$ (str): $3032.65 \mathrm{~cm}^{-1}$

$\mathrm{C}=\mathrm{N}$ (str): $2265.25 \mathrm{~cm}^{-1}, \mathrm{C}-\mathrm{O}$ (str): $1171.33 \mathrm{~cm}^{-1}$

$\mathrm{C}=\mathrm{O}(\mathrm{str}): 1736.19 \mathrm{~cm}^{-1}, \mathrm{C}-\mathrm{S}$ (Str): $2573.44 \mathrm{~cm}^{-1}$

\section{Chemical / IUPAC Name}

2-[(2 chloro-benzylidene)-amino]-6- methyl-4,5,6,7-tetrahydrothieno[2,3-c]pyridine-3-carboxylic acid ethyl ester

Infrared Spectroscopy Values

C-H (str): $2982.52 \mathrm{~cm}^{-1}, \mathrm{H}=\mathrm{C}$ (str): $3032.65 \mathrm{~cm}^{-1}$

$\mathrm{C}=\mathrm{N}$ (str): $2265.25 \mathrm{~cm}^{-1}, \mathrm{C}-\mathrm{O}$ (str): $1171.33 \mathrm{~cm}^{-1}$

$$
\begin{aligned}
& \mathrm{C}=\mathrm{O}\left(\text { str) }: 1736.19 \mathrm{~cm}^{-1}\right. \\
& \mathrm{C}-\mathrm{S} \text { (Str): } 2573.44 \mathrm{~cm}^{-1}
\end{aligned}
$$

\section{Chemical / IUPAC Name}

2-[(4 chloro-benzylidene)-amino]-6- methyl-4,5,6,7-tetrahydrothieno[2,3-c] pyridine-3-carboxylic acid ethyl ester

Infrared Spectroscopy Values

C-H (str): $2985.28 \mathrm{~cm}^{-1}, \mathrm{H}=\mathrm{C}$ (str): $3012.65 \mathrm{~cm}^{-1}$

$\mathrm{C}=\mathrm{N}$ (str): $2265.16 \mathrm{~cm}^{-1}, \mathrm{C}-\mathrm{O}$ (str): $1174.13 \mathrm{~cm}^{-1}$

\section{C-S (Str) $2579.44 \mathrm{~cm}^{-1}$,}

$\mathrm{C}=\mathrm{O}(\mathrm{str}): 1732.19 \mathrm{~cm}^{-1}$

\section{Chemical / IUPAC Name}

2-[(3,4-dimethoxy-benzylidene)-amino]-6- methyl-4,5,6,7-tetrahydrothieno[2,3-c] pyridine-3-carboxylic acid ethyl ester

Infrared Spectroscopy Values

C-H (str): $2996.26 \mathrm{~cm}^{-1}, \mathrm{H}=\mathrm{C}$ (str): $3035.65 \mathrm{~cm}^{-1}$

$\mathrm{C}=\mathrm{N}$ (str): $2278.16 \mathrm{~cm}^{-1}, \mathrm{C}-\mathrm{O}$ (str): $1184.23 \mathrm{~cm}^{-1}$

$$
\mathrm{C}=\mathrm{O}(\mathrm{str}): 1738.16 \mathrm{~cm}^{-1}
$$$$
\text { C-S (Str): } 2589.54 \mathrm{~cm}^{-1}
$$

\section{Chemical / IUPAC Name}

2-(benzylideneamino)-6-methyl-4,5,6,7-tetrahydro-thieno[2,3-c] pyridine-3- carboxylic acid ethyl ester

Infrared Spectroscopy Values

C-H (str): $2928.76 \mathrm{~cm}^{-1}, \mathrm{H}=\mathrm{C}$ (str): $3062.15 \mathrm{~cm}^{-1}$

$\mathrm{C}=\mathrm{N}$ (str): $2235.29 \mathrm{~cm}^{-1}, \mathrm{C}-\mathrm{OH}(\mathrm{str}): 3318.35 \mathrm{~cm}^{-1}$

C-O (str): $1178.23 \mathrm{~cm}^{-1}, \mathrm{C}=\mathrm{O}(\mathrm{str}): 1735.49 \mathrm{~cm}^{-1}$

C-S (Str): $2563.51 \mathrm{~cm}^{-1}$

Chemical / IUPAC Name

2-[(4-Hydroxy-3-methoxy-benzylidene)- amino]-6-methyl-4,5,6,7tetrahydro- thieno[2,3-c]pyridine-3-carboxylic acid ethyl ester

Infrared Spectroscopy Values

C-H (str): $2991.26 \mathrm{~cm}^{-1}, \mathrm{C}-0 \mathrm{H}$ (str):3314.12 $\mathrm{cm}^{-1}$

$\mathrm{H}=\mathrm{C}$ (str): $3035.65 \mathrm{~cm}^{-1}, \mathrm{C}=\mathrm{N}$ (str): $2278.16 \mathrm{~cm}^{-1}$

C-O (str): $1182.26 \mathrm{~cm}^{-1}, \mathrm{C}=\mathrm{O}(\mathrm{str}): 1735.16 \mathrm{~cm}-\mathrm{C}-\mathrm{S}$ (Str): $2591.26 \mathrm{~cm}^{-1}$ 
Table 3: Antibacterial and Antifungal activities of 2 -((substituted benzylidene) -imino)-3-carbethoxy-6-N- methyl piperidino thiophene analogues (1a-l).

\begin{tabular}{ccc}
\hline Test compounds & B. subtillis & E. coli \\
\hline S1-1j- & $39.26 \pm 0.11$ & $35.3 \pm 0.17$ \\
S1-1l & $38.2 \pm 0.50$ & $33.03 \pm 0.08$ \\
S1-1f & $35.53 \pm 0.15$ & $32.06 \pm 0.12$ \\
S1-1k & $30.16 \pm 0.07$ & $29.5 \pm 0.02$ \\
S1-1e & $29.46 \pm 0.11$ & $28.36 \pm 0.27$ \\
S1-1a & $27.5 \pm 0.16$ & $28.23 \pm 0.14$ \\
S1-1c & $25.06 \pm 0.09$ & $27.1 \pm 0.05$ \\
S1-1d & $23.96 \pm 0.09$ & $25.4 \pm 0.2$ \\
S1-1g & $23.3 \pm 0.16$ & $21.23 \pm 0.17$ \\
S1-1i & $19.06 \pm 0.09$ & $20.16 \pm 0.2$ \\
S1-1b & $18.23 \pm 0.11$ & $19.03 \pm 0.26$ \\
S1-1h & $17.1 \pm 0.12$ & $18.3 \pm 0.15$ \\
Streptomycin & $43.56 \pm 0.20$ & $39.53 \pm 0.27$ \\
\hline
\end{tabular}

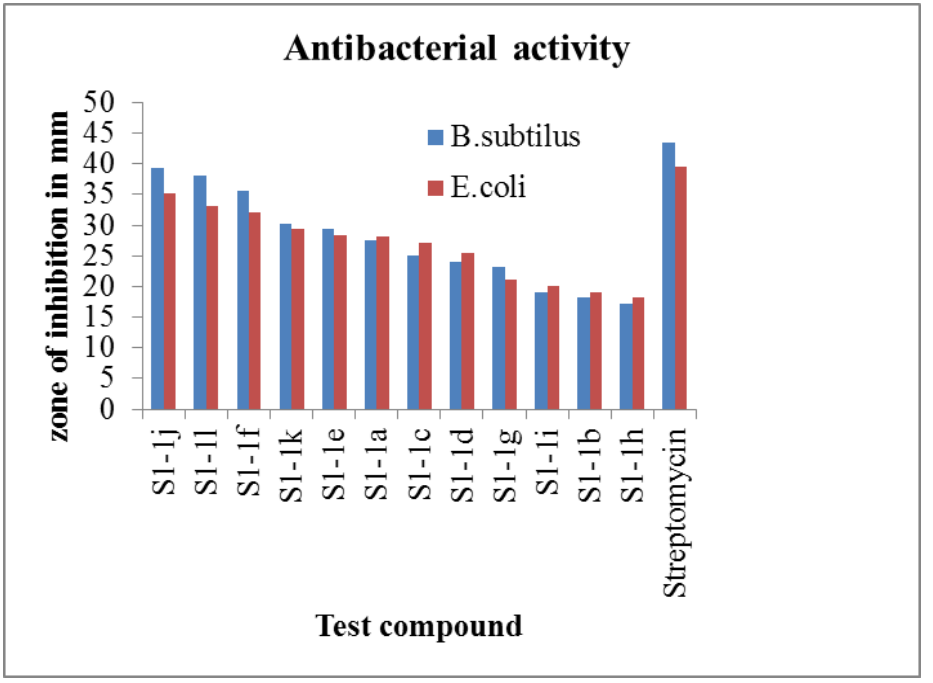

\begin{tabular}{cc}
\hline Test compounds & Aspergillus niger \\
\hline 1j & $28.56 \pm 0.2$ \\
1l & $25.56 \pm 0.08$ \\
If & $23.2 \pm 0.11$ \\
1k & $20.3 \pm 0.15$ \\
le & $18.23 \pm 0.14$ \\
la & $17.46 \pm 0.26$ \\
$1 \mathrm{c}$ & $16.06 \pm 0.12$ \\
$1 \mathrm{~d}$ & $15.36 \pm 0.27$ \\
$1 \mathrm{~g}$ & $13.2 \pm 0.17$ \\
1i & $12.4 \pm 0.26$ \\
$1 \mathrm{~b}$ & $11.03 \pm 0.08$ \\
$1 \mathrm{~h}$ & $10.26 \pm 0.14$ \\
Clotrimazole & $30.53 \pm 0.20$ \\
\hline
\end{tabular}

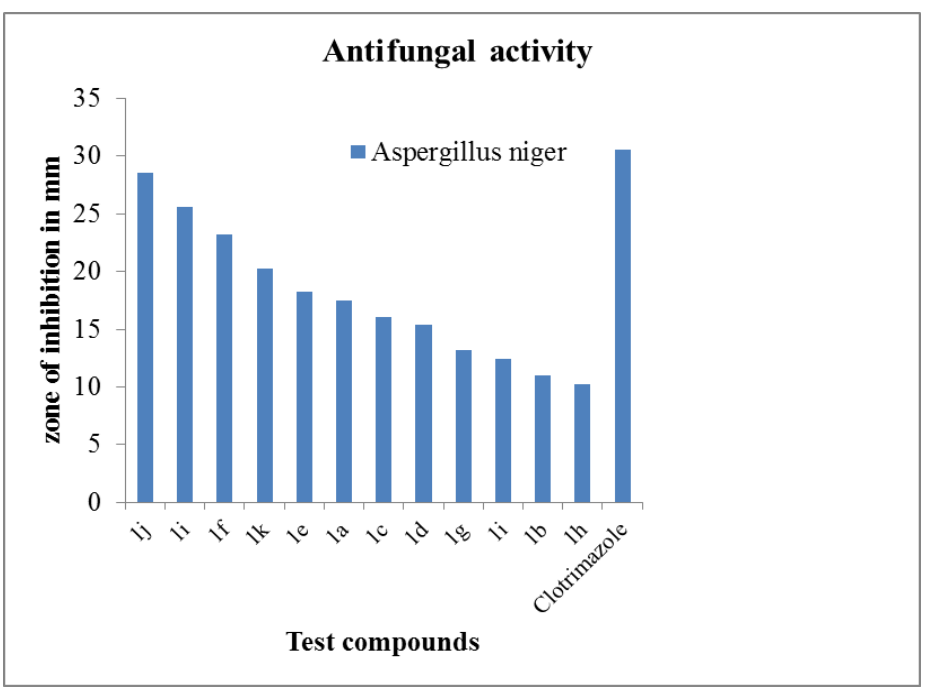

- The organisms were allowed to settle on the medium for 5 mins (seeding).

- Using a sterile cork borer $6 \mathrm{~mm}$ bore was done.

- $100 \mu \mathrm{l}$ of $100 \mu \mathrm{g}$ the test sample was transferred aseptically into the bore.

- In the same plate standard (Cefixime $1 \mathrm{mg} / \mathrm{ml}$ ) as positive control and blank (Dimethylsulphoxide) as negative control were maintained.

- $\quad$ The plates were incubated for $24 \mathrm{hrs}$ at $37^{\circ} \mathrm{C}$.

- The tests were done in triplicates.

- The zone of inhibition was measured in $\mathrm{mm}$ using standard Hi-media scale and readings were recorded.

\section{Antifungal activity}

The in vitro antifungal assay was also carried out by Kirby Baurer method. The spore of Cladosporium sps were suspended in the $5 \mathrm{ml}$ sterile distilled water and was incubated for $1 \mathrm{hr}$ at $37^{\circ} \mathrm{C}$. This $100 \mu \mathrm{l}$ spore suspension was used for the spread plate technique the media being potato dextrose agar. Using a sterile cork borer $6 \mathrm{~mm}$ bore was done. $100 \mu \mathrm{l}$ of $100 \mu \mathrm{g}$ of the test sample was transferred aseptically into the bore. In the same plate standard (Ketoconazole $1 \mathrm{mg} / \mathrm{ml}$ ) as positive

control and blank (Dimethylsulphoxide) as negative control were maintained. The plates were incubated for $24 \mathrm{hrs}$ at $37^{\circ} \mathrm{C}$. The tests were done in triplicates. The zone of inhibition was measured in $\mathrm{mm}$ using standard Hi-media scale and readings were recorded.

\section{Molecular Docking}

Molecular docking studies were carried out to predict the synthesized compounds on thymidylate synthase which is target for inhibiting the micro-organism by suppressing the de novo synthesis of pyrimidine nucleotides. The crystal structure of Thymidylate Kinase (TMK) (PDB ID 2qqe) was retrieved from Protein Data Bank and the protein structure was corrected by using Protonate 3D and energy minimization was done The ligands structures are built. The active site of protein was predicted by using site finder with default settings, dummies were assigned. The structures of the ligands were constructed and the synthesized molecules (ligands) were docked into the active site using Molecular docking software PATCH Dock with default parameters. Molecular docking of the molecules revealed the atomic contact energy (ACE) and the amino acid binding residues that are as depicted in Tables below. The precise location of the binding site and the potentiality of the ligand to bind to the active site were determined using automated docking software, that is based on guided differential evolution and a force filed based screening 


\begin{tabular}{|c|c|c|c|c|c|c|}
\hline \multirow{2}{*}{$\begin{array}{c}\text { SI no } \\
1\end{array}$} & \multirow{2}{*}{$\begin{array}{c}\text { Name of Biomarkers } \\
\text { THYMILIDATE } \\
\text { SYNTHETASE }\end{array}$} & \multirow{2}{*}{$\begin{array}{c}\text { Compound } \\
\text { Code } \\
\text { S1-1a }\end{array}$} & \multicolumn{2}{|c|}{$\begin{array}{c}\text { Details of } \\
\text { Hydrogen Bonds }\end{array}$} & \multirow{2}{*}{$\begin{array}{c}\text { ACE } \\
-190.78\end{array}$} & \multirow{2}{*}{$\begin{array}{c}\text { Amino acid residues on the docked domains } \\
\text { Arg 70, Ile 73, Glu 74,Glu 75, Arg } 102\end{array}$} \\
\hline & & & 01 & 2.868 & & \\
\hline 2 & SYNTHETASE & $\mathrm{S} 1-1 \mathrm{~b}$ & 02 & $-1.239,3.17$ & 187.87 & $\begin{array}{l}\text { Tyr 76, His 61, Ala 60, Glu 59, } \\
\text { Val 58, Lys 32, Lys 33, Glu } 74\end{array}$ \\
\hline 3 & & S1-1c & 0 & & -216.03 & Glu 74, Glu 75, Asp 76,Arg 102, Gly 103, Ile 104 \\
\hline 4 & & S1-1d & 01 & -0.5245 & -173.55 & Pro 39, Lys 40, Val 62, Glu 64,Glu 84, Phe 87, Val 163 \\
\hline 5 & & S1-1e & 02 & $-2.5,3.793$ & -161.94 & Glu 59, Ala 60, Asp 83, Thr 17,Leu 21, Lys 38, Val 58 \\
\hline 6 & & S1-1f & 01 & -2.5 & -233.43 & Phe 87 , Asn 89 , Pro 39 , Lys 40 , Glu 64 , Phe 88 \\
\hline 7 & & S1-1g & 02 & $1.90,1.78$ & -218.92 & Thr 132, Val 133, Val 133, Lys 135, Ile 134 \\
\hline 8 & & S1-1h & 04 & $\begin{array}{l}4.21,-2.33 \\
3.56,-2.48\end{array}$ & -171.56 & $\begin{array}{l}\text { Lys } 28 \text {, Lys } 33 \text {, Val } 34 \text {, Val } 56 \text {, } \\
\text { Glu } 59 \text {, His } 61 \text {, Tyr } 72 \text {, Glu } 74\end{array}$ \\
\hline 9 & & S1-1i & 0 & -- & -216.64 & Arg 70, Glu 74, Glu 75, Asp 76,Arg 76, Arg 102, Ile 104 \\
\hline 10 & & S1-1j & 01 & -0.987 & -264.47 & Gln 86, Phe 87, Pro 90, Thr 114Pro 119, Phe 120, Thr 122 \\
\hline 11 & & S1-1k & 0 & -- & -194.00 & Gly 103, Ile 104, Glu 75,Asp 76, Arg 102 \\
\hline 12 & & S1-11 & 04 & $\begin{array}{c}-2.19,-1.57 \\
0.79,-0.77\end{array}$ & -346.32 & $\begin{array}{l}\text { Phe 93, Glu 121, Phe 87, Phe 88Asn 118, Asn 89, Pro 119, Phe 120, Ile } \\
\text { 161, Asp 162, Glu } 121\end{array}$ \\
\hline
\end{tabular}

function. With the help of clustering methods, the possible binding conformations and orientations were determined. The enzyme was visualized using the sequence option. The binding site was calculated within a spacing range so that the binding site was well into the grid and interactions were analyzed using detailed energy estimates. The software was utilized to identify hydrogen bonds and hydrophobic interactions between residues at the active site and the ligand. The corresponding results were tabulated in Table 4.

\section{RESULTS}

The new thiophene analogues were screened for their antifungal and antibacterial activity ${ }^{16}$ against some of the pathogenic bacteria and fungi viz. Bacillus subtillis, Escherichia coli and Aspergillus niger. The results of investigation have been presented in Table 3 and observed that some of the compounds such as $1 \mathrm{a}, 1 \mathrm{~b}, 1 \mathrm{c}$ and $1 \mathrm{k}$ shown more activity and matching with the results of docking studies and compound $1 \mathrm{~d}, 1 \mathrm{e}, 1 \mathrm{f}, 1 \mathrm{~g}, 1 \mathrm{i}, 1 \mathrm{j}$ and 11 shown moderate activity and compound $1 \mathrm{~h}$ shown less biological activity than standard.

The docking results showed that methoxy containing compound might be more active as compared to other substituted containing compound as shown in Figure 1. The Docking studies against thymidylate kinase done to confirm the computational predictions in the Table 3. Hence, the methoxy group incorporation into the molecular structure results in greater biological activity than the other analogue, leading to an improvement in the biological activity.

\section{DISCUSSION}

The aim of designing and synthesizing new molecules as antimicrobials is because of predominant resistance of bacteria and fungi against the existing drugs. Both Piperidine and thiophenes are the heterocyclic compounds that are abundant in nature and have exhibited varied biological activity. This encouraged us to synthesize fused piperidino thiophene derivatives using established Gewald's Reaction and Schiff's Reaction and evaluate the antibacterial and antifungal activity. The
Molecular docking is employed here as an important tool in search of lead compounds of this work.

\section{CONCLUSION}

We have synthesized a series of twelve new Schiff bases containing piperidinothiophenes (1a-l) adapting standard protocols. Molecular docking studies were also carried out for all the new piperidinothiophenes (1a-1) and screened for their antifungal and antibacterial activity against some of the pathogenic bacteria and fungi viz. Bacillus subtillis, Eschochia coli and Aspergillus niger.

Some of the compounds like $1 \mathrm{j}, 1 \mathrm{l}$ and $1 \mathrm{f}$ with methoxy substituents on them shown good activity against both bacteria and fungi employed.

\section{ACKNOWLEDGEMENT}

I would like to express my sincere gratitude to my Guide and my University Dayananda Sagar University for their continuous support.

\section{CONFLICT OF INTEREST}

The authors declare no conflict of interest.

\section{ABBREVIATIONS USED}

TLC: Thin Layer Chromatography; MP: Melting Point; R Values: Gas constant; IR: Infra red; UV: Ultraviolet; NMR: Nuclear Magnetic Resonance; MS: Mass Spectroscopic; CHN: Carbon hydrogen and nitrogen; FTIR: Fourier-transform infrared spectroscopy; $\mathrm{KBr}$ : Potassium bromide; H1NMR: Hydrogen-1 NMR; CdCl: Cadmium Chloride; DMSO: Dimethyl Sulfoxide; E. coli: Escherichia coli; B. subtilis: Bacillus subtilis; TMK: Thymidylate Kinase; PDB: Protein DATA Bank; ACE: Atomic Contact Energy, viz- that is to say.

\section{REFERENCES}

1. Ajay DP, Parendu DR, Franklin PX, Harish P, Vasudevan S, Kamala KV. Synthesis of Tetra substituted thiophenes as anti-inflammatory agents. Bioorg. Med Chem. 2005;13(24):1275-83. 
2. Chung-Kyu R, Su-Kyung L, Ja-Young H, Ok-Jai J, Jung YL, Seong HJ. Synthesis and antifungal activity of 5-arylamino-4,7-dioxobenzo[b] thiophenes. Bioorg Med Chem Lettr. 2005;15(10):2617-20

3. Isabel CFRF, Rcardo CC, Leticia ME, Maria-Joao QRP. Screening of antimicrobial activity of diary amine in the 2,3,5-trimethyl benzo [b] thiophene series: A structureactivity evaluation study. Bioorg Med Chem Lettr. 2004;14:5831-3.

4. Saravanan J, Mohan S, Nargund L, Shishoo C. Synthesis of some benzo (b)thiophenes as potential anti-microbial agents. Indian J Hetero Chem. 1997;6:203-6.

5. Pier GB, Maria GP, Jhon CS, Allan RM, Valeria I, Pier AB. Synthesis of 2-amino-3hetero aroyl thiophenes and of their activity as potential allosteric enchancers at the human A1 receptor. Eup J Med Chem. 2004;39(10):855-65.

6. Henning L andra Z, Heidi F, Joel L, Ray AO, Seammells PJ. Synthesis of 2-amino-3-benzyl thiophene an allosteric enhancers of $A$ adenosine agonist binding: New 3-4-and5-modifications. J Med Chem. 2003;46(10):1870-7.

7. Saravanan J, Mohan S. Synthesis of some 2-amino-3-(N-tolyl carboxamido)4,5-pentamethylene thiophenes as potential antibacterial agents. Asian J Chem. 2003;15:625-8

8. Mohan S, Saravanan J. Synthesis, characterization and antibacterial activity of some Schiff bases of 2 - amino-3-(N-Tolyl Carboxamide)-4,5,6,7- tetrahydro benzo [b] thiophenes. Asian J Chem. 2003;15(1):67-70.

9. Tehranchian S, Akbarzadeh T, Fazeli M, Jamalifar $H$. Synthesis and anti-bacterial activity of 1- [1,2,4-triazol-3-yl] and 1-[1,3,4-thiadiazol-2-yl]-3-methylthio-6,7 dihydro-benzo[c]thiophen-4[5H]ones. Bioorg Med Chem Lettr. 2005;15(4):1023-5.

10. Biswa RP, Mohan S, Saravanan J. Synthesis, Characterization and Biological Screening of Some New Condensed N-Methyl Piperidino Thiophenes. Asian J of Chem. 2007;19(6):4368-72.

11. Anand AG, Parameshwarappa BS, Ila H. Sequential One-Pot Synthesis of Tri- and Tetrasubstituted Thiophenes and Fluorescent Push-Pull Thiophene Acrylates Involving (Het)aryl Dithioesters as Thiocarbonyl Precursors J Org Chem. 2015:80(1):414-27.

12. Gewald K. Angew. Chem. 1961;73:114.

13. Yin G, Wang Z, Chen A, Gao M, Wu A, Pan Y. The Efficient One-Pot Synthesis of Tetraalkyl Substitutes Furans from Symmetrical Acetylenes. EtAICl 2 and Carboxylic Esters Catalyzed by Cp 2 TiCl 2. J Org Chem. 2008;73:3377.

14. Minetto G, Raveglia LF, Sega A, Taddei M. Eur J Org Chem. 2005:5277.

15. Joule JA, Mills K. Heterocyclic Chemistry, Wiley: New York. 2011.

16. Kavanagh F. a) Analytical microbiology. 1963;403. (b) Burdon KL. Introduction to Microbiology, McMillan Company, New York. 1068;102.

Article History: Submission Date : 31-03-2020; Revised Date : 14-04-2020; Acceptance Date : 09-05-2020.

Cite this article: Ramalingam A, Saravanan J. Synthesis, Docking and Antimicrobial Activity Studies of Some Novel Fused thiophenes of Biological Interest. J Young Pharm. 2020;12(2):118-24. 